\title{
Emotions as Social Information in Shared Decision-Making in Oncology
}

\author{
Theresa Treffers ${ }^{a, b}$ Paul Martin Putora ${ }^{c, d}$ \\ ${ }^{a}$ Seeburg Castle University, Seekirchen am Wallersee, Austria; ${ }^{\mathrm{b}}$ TUM School of Management, Technical University \\ of Munich, Munich, Germany; ' Department of Radiation Oncology, Kantonsspital St. Gallen, St. Gallen, Switzerland \\ ${ }^{\mathrm{d}}$ Department of Radiation Oncology, Inselspital Bern, University of Bern, Bern, Switzerland
}

\section{Keywords}

Emotions · Shared decision-making · Oncology · Decision criteria $\cdot$ Treatment options · Cancer

\begin{abstract}
Emotions play an important role in decision-making and they can impact individual as well as shared decisions. With increasing complexity of the decision, the potential for emotions to influence the outcome increases. Emotions are thus an influential factor in oncological decision-making which is a complex and high-stakes situation. As the shared decisionmaking process is at the center of patient-centric decisions, we model emotions as social information that inform the shared decision-making process. We present and explain a range of emotional concepts, together with a specific clinical example, that can impact the shared decision-making process. Our process model shows that emotions are experienced in various combinations before, during, and after a shared decision is made and how patients' and physicians' emotions interact and spill over during a shared decision situation. Overall, our process model and specific example show how emotions can impact shared decision-making in oncology in a multitude of ways. With this paper, we want to raise awareness of the role of emotions in the shared decision-making process, as emotions are often not explicitly recognized as decision criteria. Increased awareness of emotions may help their optimal utilization and reduce their influence as a bias in shared decision-making.
\end{abstract}

(c) 2020 S. Karger AG, Basel

\section{Introduction}

Emotions and Decision-Making

Our emotions affect how we perceive, process, and present information, which in turn informs our decisions. Behavioral science demonstrated that intense emotional events such as natural disasters $[1,2]$, terrorist attacks [3, 4], violent trauma $[5,6]$, or financial crises [7] impact individuals' decisions. However, even minor daily events, such as rainy or sunny weather [8-10], have been shown to influence individuals' emotions and, in turn, their decision-making process. Emotions particularly inform our decision-making process when we make complex decisions [11]. This is counterintuitive because one could assume that the more time we take to collect and process information to decide, the more rational the decision becomes. It is exactly the opposite: complex decision situations need substantive information processing, which takes time, and which gives emotions more opportunity to influence the decision. This results in decisions that are rationalized in an emotional sense (i.e., satisfying for the decision maker) rather than an economical one (i.e., a decision with higher payoffs than costs).

Applying insights from social psychology, economic scholars have recognized that people systematically deviate from rational decision-making, i.e., decisions that follow the principles of the economic decision-making model of the "homo oeconomicus". These deviations can be traced back to a number of "human" factors such as

Theresa Treffers

Seeburg Castle University

Seeburgstrasse 8

AT-5201 Seekirchen am Wallersee (Austria)

E-Mail theresa.treffers@uni-seeburg.at 


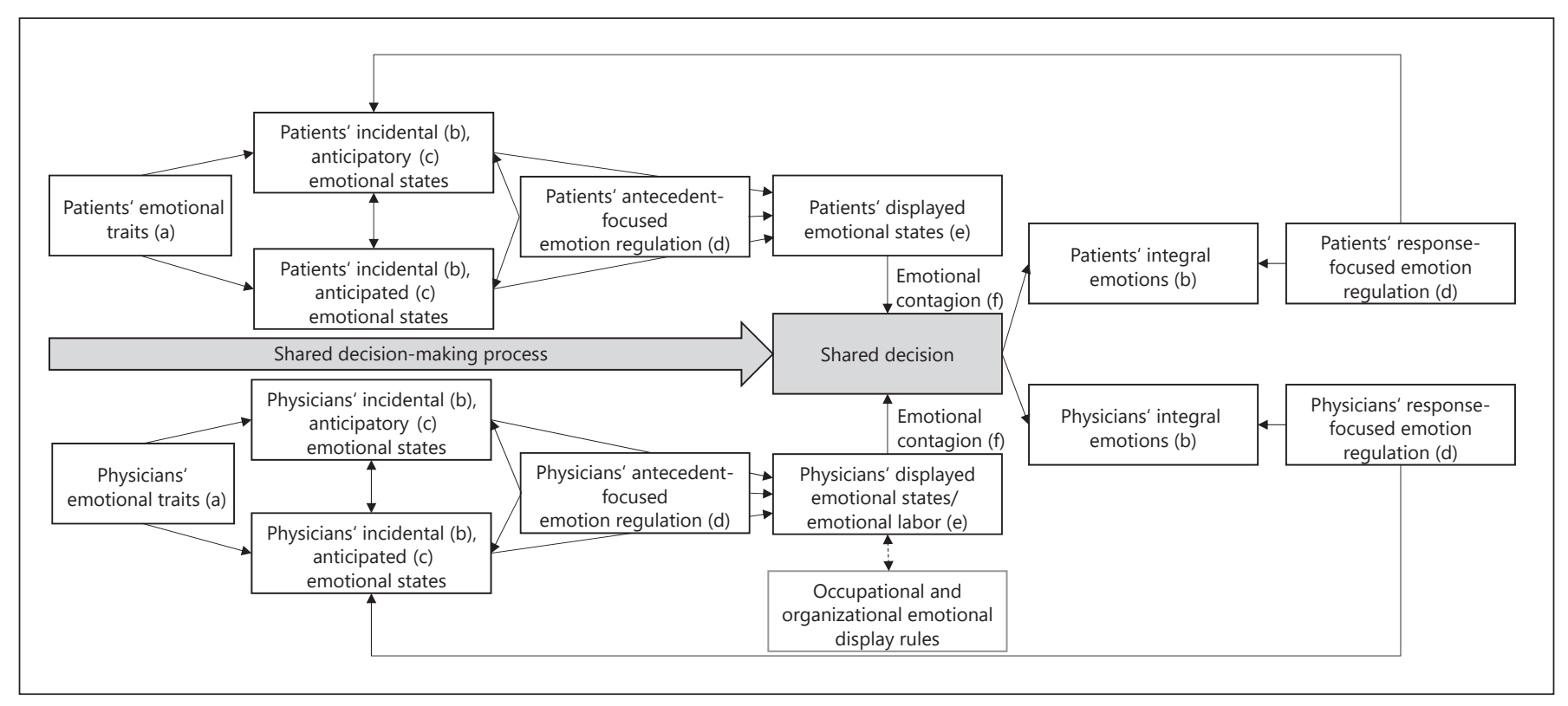

Fig. 1. Interpersonal model of emotions in shared decision-making.

emotions or cognitive biases that are not considered in the classic economic model $[12,13]$. In this paper, we address emotions as information in the shared decisionmaking process in oncology.

\section{Shared Decision-Making in Oncology}

In oncology, many factors lead to the availability of multiple treatment options for a single disease and situation. For example, multiple drugs, often from different pharmaceutical companies, can be available for the same disease or similar outcomes can be achieved with very different modalities (e.g., surgery or radiotherapy $[14,15]$ ). In oncology, many decisions can be considered highstakes and a variety of decision criteria can be called upon to make a recommendation on the physician's side and a decision on the patient's side [16]. Hence, oncological decision-making is complex and requires substantive processing which gives rise to emotional, and thus potentially biasing, influences.

In recent decades a shift has taken place in oncological decision-making from a rather authoritative approach of physicians dominating treatment decisions to a stronger involvement of the patient to reach a shared decision. In shared decision-making, the physician provides the patient with all available information and helps guide what is ultimately the patient's decision. In theory, this sounds significantly better than the previous approach, yet it "as- sumes that patients are capable of taking in complex information from health care providers at a most vulnerable time in their lives and then synthesizing that information to make rational treatment choices" [13]. The complexity of information involved in oncological decision-making has led to the implementation of decision aids in oncology [17]. While their dissemination is still limited, multiple trials have demonstrated positive impacts on the decision-making process. Their use has been shown to increase the patient's contribution to the final decision and reduce decisional conflict [18].

Extant literature on shared decision-making has started to investigate the influence of cognitive biases [13] and emotions $[19,20]$, but, to the best of our knowledge, no paper has focused on a range of emotional concepts and their influences along the shared decision-making process. This paper addresses this shortcoming and aims to provide a conceptual model of how a variety of emotional concepts can influence the shared decision-making process in oncology. As evident from the term shared decision-making, the patient is not the only one involved. The physician (or any other health care worker involved in the decision process) is equally relevant in this process and is also susceptible to various emotional influences and biases. Hence, our aim was to develop an interpersonal model of emotions that outlines how emotions may inform the shared decision-making process involving the 
patient and the physician (see Fig. 1). Knowing how and when emotions can impact the patient and the physician along the shared decision-making process may help improve the final decision and ultimately contribute to better outcomes. When presenting our process model, we also provide a specific clinical example (i.e., a patient with prostate cancer choosing between active surveillance and active treatment) to illustrate the clinical relevance of how different emotional concepts can impact the shared decision-making process in several instances.

Concepts like risk and uncertainty are inherent within the shared decision-making process. While risky decision situations contain objective probabilities about possible outcomes, uncertain situations miss out on precise, probabilistic information about decision outcomes [21]. Although in practice, risk and uncertainty are often dealt with in a similar way, they are only weakly related and describe distinct concepts. Social science is not consistent with this distinction, hence empirical evidence about how emotions impact decisions under risk and uncertainty is mixed. In general, research has shown that emotions can influence individual risk preferences [22-25], but many studies have reached conflicting conclusions and provide different explanations for their findings. Research on emotions and decisions under uncertainty is scarce [26], but also suggests that emotions can impact individual uncertainty preferences. In this paper, while acknowledging that emotions can influence concepts like risk and uncertainty within shared decision-making, we focus on the role of emotional concepts in the overall shared decisionmaking process.

\section{Emotions as Social Information in Shared Decision- Making}

\section{Emotions as Social Information}

Psychological science has a long tradition in studying the intrapersonal function of emotions for decision-making. A very prominent theory about how emotions can influence a person's judgment and decision-making process is the affect-as-information theory. Affect-as-information theory proposes that affective cues of emotional states directly influence judgments by providing experiential and bodily information that is attributed to the object of judgment $[27,28]$. Thus, when individuals evaluate a decision situation, they may mistake pre-existing feelings as a reaction to the situation and use this emotion as information when deciding. In our clinical example, a patient may experience sadness due to the received diagno- sis of prostate cancer which in turn can influence his/her perception of side effects during an appointment in the hospital.

While affect-as-information describes how emotions can inform a person's decision-making process intrapersonally, behavioral science has recently started to add the interpersonal function of emotions [29-31] - known as social-functional theories of emotions. Following the emotions as social information theory [31,32], we argue that physicians and patients do not just experience their emotions, but also express them to others. The outward expression of emotions triggers mutual affective reactions and thoughts, which in turn shape the judgments and decisions of both. In our clinical example, the physician would perceive the patient's sadness during their appointment in the hospital and react to it.

\section{Defining Affect, Emotion, Mood, and Feeling}

Behavioral studies investigating emotions have often used the terms affect, emotion, and moods interchangeably. Since recently, there is an agreement on how to distinguish these terms [33]: affect is often regarded as an umbrella term that spans across a broad range of emotional experiences and studies often refer to general positive and negative affect. In contrast to affect, emotions are short-lived, intense feelings that are directed at a certain object. Moods, compared to emotions, are longer-lived, mild, diffuse feelings without a clearly identifiable cause. Some of these negative moods may arise during winter and are described as seasonal affective disorders (SAD or winter blues) [34].

In our clinical example, a prostate cancer patient could feel calm in the presence of a physician or be afraid of the test result (emotion). However, the patient may also feel worried for weeks during treatment, even though the treatment is going according to schedule and is well tolerated; hence, the source of the patient's worry is less specific and not directly caused by the treatment (mood). All these emotional experiences can be summarized as the patient's affect.

\section{Emotional Dimensions and Discrete Emotions}

When defining emotional states, two concepts have emerged that are not mutually exclusive, but rather complementary. First, the dimensional model of emotion describes emotions as consisting of two underlying dimensions, i.e., valence and arousal. Valence represents the hedonic tone as a continuum of pleasure and displeasure, while arousal represents the level of bodily activation [35]. 


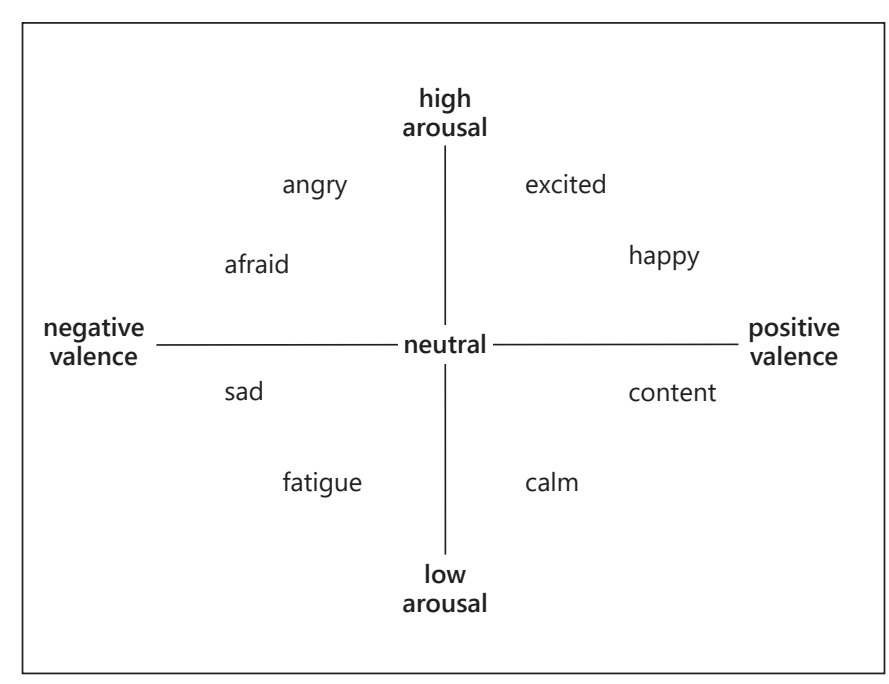

Fig. 2. Valence and arousal dimensional model for some basic emotions.

Second, discrete emotion theory builds on the concept of basic or discrete emotions in that they are assumed to be unique experiential states that stem from distinct causes [36]. Depending on the researchers, the number of discrete emotions varies and could include interest, joy, surprise, sadness, anger, disgust, contempt, self-hostility, fear, shame, shyness, and guilt [37]. Basic emotions are assumed to be present from birth, are experienced regularly, and span across all cultures [38]. Figure 2 shows a valencearousal dimensional model for some basic emotions.

Constructs like love, hate, envy, or passion are not considered basic emotions as a considerable social and cognitive component is required to form these emotional experiences. In addition, it usually takes more than one situation to experience these emotions whereas discrete emotional states can arise from a single instance.

In our clinical example, a prostate cancer patient may experience strong displeasure (high arousal, negative valence) during his treatment. Similarly, a patient with prostate cancer could experience discrete anger because his treatment is not effective or discrete surprise because the treatment is overly effective. Finally, when a physician is responsible for a patient for many years during his/her treatment, the patient can develop a deep emotional (positive or negative) relationship with the physician.

\section{Emotional States and Emotional Traits}

Within emotion research, we can distinguish between emotional states and emotional traits [39] (Fig. 1a). While emotional states describe the current emotional feeling in a specific situation, emotional traits represent rather stable personality traits, which, for example, describe a tendency to frequently feel happy or sad. While emotional traits largely determine the tendency of emotional states a person experiences in a range of situations, these may deviate from emotional states.

In our clinical example, imagine that a patient and a physician have to decide between treatment (e.g., prostatectomy or radiotherapy) and active surveillance for a low-risk prostate cancer. The patient might be a rather skeptical and pessimistic person (negative emotional trait), while the physician may be a person who is typically in a good mood and has, what her/his friends describe as, a "positive personality" (positive emotional trait). Even though the patient has a negative emotional trait and tends to experience negative emotional states, he may experience positive emotional states (e.g., contentment) after he has discussed treatment and active surveillance with the physician.

\section{Incidental and Integral Emotions}

Emotion research also distinguishes between incidental and integral emotional states [40] (Fig. 1b). Incidental emotions are not evoked by the current decision but are independently experienced from the situation. In contrast, integral emotional states are induced by the current decision situation, which is the shared decision about treatment for prostate cancer in our model.

Social emotions are related to the concept of integral emotions [41]. Social emotions are emotions that depend on social interaction, particularly the emotions, cognitions, and behaviors of other people. That is, people can only feel embarrassment, empathy, or pride in the presence of others in contrast to sadness, for example, which is experienced independent of others' emotions, cognitions, and behavior. Furthermore, people can only feel guilty, jealous, or empathic in reference to another subject.

In our clinical example, driving a sports car to work on a sunny day might evoke incidental happiness, which could potentially influence a physician's treatment decision in an optimistic way (e.g., interpret decision criteria for a prostate cancer patient in a rather positive light), even though his incidental happiness is not related to the actual treatment recommendation. In contrast, when a physician makes a shared decision with a prostate cancer patient, this decision may evoke integral pity within the physician. Finally, a physician treating a prostate cancer patient successfully, even though the prospects for recovery are very bad, can display pride only in the presence of the patient, but not with herself/himself. 


\section{Anticipatory and Anticipated Emotions}

During shared decision-making, physicians and patients can be influenced by the emotions they currently experience (i.e., anticipatory or immediate emotions) but also by the emotions they expect to experience after the shared decision (i.e., anticipated or expected emotions). That is, anticipated emotions are expectations of how a person will feel once gains or losses associated with a decision are experienced [42] (Fig. 1c).

Again taking our specific clinical example, imagine that the physician had a very stressful week - next to routine work and family chores, several deadlines for grants and papers are due this week - setting him in a rather negative and stressed mood (anticipatory/immediate emotion). In addition, the physician expects to be excited after the decision situation because she/he was recently involved in a scientific analysis of data related to active surveillance for prostate cancer and is looking forward to applying his newly refreshed knowledge (anticipated/expected emotion). The patient may experience anger, because his primary physician had initially told him that she/he does not have cancer and now she/he needs to meet with another physician to discuss cancer treatment options (anticipatory/immediate emotion). Additionally, the patient may expect to feel fear after the decision situation as she/he had read disturbing reports of side effects of cancer treatments (anticipated/expected emotions).

\section{Emotion Regulation}

The regulation of emotion refers to processes that influence which emotions are experienced, when they are experienced, and how they are experienced or expressed [43] (Fig. 1d). There are different emotion regulation strategies that can be applied throughout the shared decision-making process [43]. Antecedent-focused emotion regulation occurs before the emotion is generated and focuses on manipulating the emotional cues that would generate an emotion; response-focused emotion regulation occurs after the emotion has been generated and focuses on manipulating the emotional responses [44].

Emotions can be regulated within a person - intrinsic emotion regulation - or a person seeks to regulate emotions in someone else - extrinsic emotion regulation [45]. Furthermore, research differentiates between a person's motivation to engage in emotion regulation. This motivation can either be hedonic (i.e., feel less negative or more positive in the near term) or instrumental (i.e., achieve one's long-term goals) [46]. Finally, the emotion regula- tion goal can be explicit or implicit (e.g., if the goal is explicit, the goal is deliberate and consciously perceived; if the goal is implicit, the goal is activated outside of a person's awareness) [47].

Returning to our clinical example, the physician could regulate incidental fatigue and contentment (i.e., emotions that are experienced before entering the decision situation), while the patient regulates his incidental fear and anger (antecedent-focused emotion regulation). Specifically, the physician may apply intrinsic emotion regulation when trying not to look too tired (intrinsic emotion regulation), while also extrinsically trying to regulate the patient's fear and anger. In addition, the physician may choose to regulate her/his fatigue to feel better after an appointment with the patient (hedonic emotion regulation), while also regulating her/his fatigue to prevent a burnout (instrumental emotion regulation). Finally, the goal of the physician's emotion regulation strategy is explicit when she/he tries to look happier than she/he actually feels in front of a patient with prostate cancer; or the goal is implicit when the physician tones down emotional responses (such as speaking softly or neutralizing mimics) without knowing she/he is trying to do so when entering a difficult conversation with a patient, e.g., confronting the patient with a cancer diagnosis. After the decision situation, the physician could upregulate his integral emotions (i.e., the emotions that are elicited as a result of the shared decision situation) by sharing his new-found knowledge with colleagues to receive positive confirmation, while the patient downregulates his integral negative emotions (response-focused emotion regulation).

\section{Emotional Display and Emotional Labor}

Emotions can be displayed in a person's face, voice, and actions that are perceived to reflect the observed individual's underlying emotions. While patients may display their emotions openly in a shared decision-making process, physicians may not be as open due to their perception of professional codes. In this case, the physician's displayed emotions do not reflect the experienced emotions, which is termed emotional labor [48] (Fig. 1e).

Emotional labor is described as the effort, planning, and control needed to express occupationally or organizationally desired emotions during interpersonal transactions. In occupational psychology, emotional labor has often been investigated as an antecedent of emotional exhaustion and burnout. These studies show that continuous emotional labor weakens one's individual capacity to deal with other emotional challenges which ultimately 
leads to the feeling of not having available resources to compensate further burden and being burnt-out $[49,50]$.

In our clinical example, the patient may be very authentic in displaying his frustration to the physician in the decision situation (emotional display), while the physician, who had a busy and stressful workday, has learned to appear calm and organized when she/he speaks to the patient (emotional labor).

\section{Emotional Contagion}

When expressing emotions in the shared decisionmaking process, physicians and patients can be influenced by each other's emotions, which is known as emotional contagion. Emotional contagion often occurs at a less conscious level, based on automatic fast process of automatic, continuous, synchronous nonverbal mimicry and feedback [51] (Fig. 1f).

In our clinical example, a physician's enthusiasm about the success of a treatment during shared decisionmaking may spill over to the patient and turn his fear of dying into hope of surviving (emotional contagion). In addition, the patient's negative displayed emotions may spill over to the physician during shared decision-making (emotional contagion). The physician can then experience integral fatigue and worry after answering unexpected questions and as she/he felt the patient challenged his/ her new-found knowledge.

\section{Discussion}

Extant research in the behavioral and medical sciences has made important advances by showing how sources of biases, such as emotions, can impact individual and social decision-making. Building on this research, we sought to present a model that illustrates emotional influences throughout the shared decision-making process. The shared decision-making process is of peculiar importance as patients are increasingly involved in treatment decisions. Due to its social nature, we model emotions as social information that are incorporated into the decision-making process between physician and patient. Our clinical example demonstrates how the impact of emotions can specifically influence the shared decision-making process for a patient with low-risk prostate cancer choosing between active surveillance and treatment. Our model and clinical example show that emotional influences on the decision-making process are not linear, but complex and intertwined.

It is challenging to empirically examine comprehensive models such as ours and to grasp all possible influ- ences that emotions can have on the shared decisionmaking process. Nevertheless, we recognize the need for future research to empirically test our model step by step and seek to make some recommendations that we deem particularly important for empirical investigation. First, we believe that the interactive influence of emotional state and trait throughout the shared decision-making process is worthy of further research. While emotional traits are rather stable personality traits and can hardly be adapted, emotional states can be measured and explicitly (i.e., directly) or implicitly (i.e., subliminally) shaped to positively influence the shared decision-making process and subsequent treatment. Second, we recommend future research to investigate emotion regulation strategies before the shared decision situation to reach decisions that are aligned with optimal treatment success and personal desires, as well as after the shared decision to prepare the patient emotionally for the actual treatment trajectory. Such studies could be instrumental in advising physicians how to actively regulate their own and the patients' emotions. Third, an empirical investigation of displayed emotions in the shared decision situation, particularly including physicians' display rules and their emotional labor, would be beneficial as a basis for emotional guidance for physicians in the shared decision situation.

While there is more to investigate in the field of emotions and clinical decision-making, we hope that we have raised awareness about this topic by showing possible emotional influences along the shared decision-making process. Emotions are essential for making decisions, but they can also (consciously or subconsciously) bias decisions. Medical science and practice has started acknowledging this challenge and has developed several important advances to improve patient-oriented clinical decision-making. This includes, for example, the increased use of patient-reported outcomes [17, 18, 52], which have been shown to increase the implementation of shared decision-making and can serve as a basis for clinical decision support systems [53]. Another approach that has been shown to improve decision-making is the use of patient decision aids $[17,18]$.

Finally, in the emerging era of big data [54], large clinical databases are increasingly used to provide insights into decision-making; however, these databases typically do not encode the participants' or the physicians' emotions. This is an area where self-reported outcomes can potentially provide information on emotions, which would otherwise not be measurable. Further information may be provided by machine learning algorithms tapping into new sources of information, such as online cancer 
support groups for example $[55,56]$. Our model might help position this input within the shared decision-making process.

\section{Conclusion}

Our model of emotions as social information in shared decision-making emphasizes the interpersonal influence emotions can have in the oncological decision-making process. Our awareness of the influence of emotions may help us better understand how decisions are made and may also help us understand the psychological complexity leading to a variety of answers to the same oncological problem.

\section{Disclosure Statement}

The authors have no conflicts of interest to declare.

\section{Funding Sources}

The authors have no funding sources to declare.

\section{Author Contributions}

The authors contributed equally to this paper.

\section{References}

1 Eckel CC, El-Gamal MA, Wilson RK. Risk loving after the storm: A Bayesian-Network study of Hurricane Katrina evacuees. J Econ Behav Organ. 2009;69(2):110-24.

2 Botzen WW, de Boer J, Terpstra T. Framing of risk and preferences for annual and multiyear flood insurance. J Econ Psychol. 2013;39: 357-75.

3 Sunstein CR. Terrorism and probability neglect. J Risk Uncertain. 2003;26(2-3):121-36.

4 Viscusi WK. Valuing risks of death from terrorism and natural disasters. J Risk Uncertain. 2009;38(3):191-213.

5 Voors MJ, Nillesen EE, Verwimp P, Bulte EH, Lensink R, van Soest DP. Violent conflict and behavior: a field experiment in Burundi. Am Econ Rev. 2012;102(2):941-64.

6 Callen M, Isaqzadeh M, Long JD, Sprenger C. Violence and risk preference: experimental evidence from Afghanistan. Am Econ Rev. 2014;104(1):123-48.

7 Guiso L, Sapienza P, Zingales L. Time varying risk aversion. J Financ Econ. 2018;128(3): 403-21.

8 Hirshleifer D, Shumway T. Good day sunshine: stock returns and the weather. J Finance. 2003;58(3):1009-32.

9 Kliger D, Levy O. Mood-induced variation in risk preferences. J Econ Behav Organ. 2003; 52(4):573-84.

10 De Silva DG, Pownall RA, Wolk L. Does the sun 'shine'on art prices? J Econ Behav Organ. 2012;82(1):167-78.

11 Forgas JP. Mood and judgment: the affect infusion model (AIM). Psychol Bull. 1995 Jan; 117(1):39-66.

12 Blumenthal-Barby JS, Krieger H. Cognitive biases and heuristics in medical decision making: a critical review using a systematic search strategy. Med Decis Making. 2015 May;35(4):539-57.

13 Ozdemir S, Finkelstein EA. Cognitive bias: the downside of shared decision making. JCO Clin Cancer Inform. 2018 Dec;2(2):1-10.
14 Panje CM, Glatzer M, Sirén C, Plasswilm L, Putora PM. Treatment Options in Oncology. JCO Clin Cancer Inform. 2018 Dec;2(2):1-10.

15 Putora PM, Leskow P, McDonald F, Batchelor $\mathrm{T}$, Evison M. International guidelines on stage III N2 non-small cell lung cancer: surgery or radiotherapy? ERJ Open Res. Forthcoming 2020.

16 Glatzer M, Panje CM, Sirén C, Cihoric N, Putora PM. Decision making criteria in oncology. Oncology. 2018. DOI: 10.1159/000492272.

17 Ankolekar A, Dekker A, Fijten R, Berlanga A. The benefits and challenges of using patient decision aids to support shared decision making in health care. JCO Clin Cancer Inform. 2018 Dec;2(2):1-10.

18 McAlpine K, Lewis KB, Trevena LJ, Stacey D. What is the effectiveness of patient decision aids for cancer-related decisions? A systematic review subanalysis. JCO Clin Cancer Inform. 2018 Dec;2(2):1-13.

19 Lee RT, Lovell BL, Brotheridge CM. Relating physician emotional expression to shared understanding and shared decision-making with patients. Int J Work Organ Emot. 2010; 3(4):336-50.

20 Dicé F, Dolce P, Freda MF. Exploring emotions and the shared decision-making process in pediatric primary care. Mediterr J Clin Psychol. 2016;4(3).

21 Knight FH. Risk, uncertainty, and profit. Boston: Hart, Schaffner \& Marx; 1921.

22 Loewenstein GF, Weber EU, Hsee CK, Welch N. Risk as feelings. Psychol Bull. 2001 Mar; 127(2):267-86.

23 Anderson CJ. The psychology of doing nothing: forms of decision avoidance result from reason and emotion. Psychol Bull. 2003 Jan; 129(1):139-67.

24 Pham MT. Emotion and rationality: A critical review and interpretation of empirical evidence. Rev Gen Psychol. 2007;11(2):155-78.
25 Lerner JS, Li Y, Valdesolo P, Kassam KS. Emotion and decision making. Annu Rev Psychol. 2015 Jan;66:799-823.

26 Baillon A, Koellinger PD, Treffers T. Sadder but wiser: the effects of emotional states on ambiguity attitudes. J Econ Psychol. 2016;53: 67-82.

27 Schwarz N, Clore GL. Mood, misattribution, and judgments of well-being: informative and directive functions of affective states. J Pers Soc Psychol. 1983;45(3):513-23.

28 Clore GL, Huntsinger JR. How emotions inform judgment and regulate thought. Trends Cogn Sci. 2007 Sep;11(9):393-9.

29 Côté S. A social interaction model of the effects of emotion regulation on work strain. Acad Manage Rev. 2005;30(3):509-30.

30 Sy T, Côté S, Saavedra R. The contagious leader: impact of the leader's mood on the mood of group members, group affective tone, and group processes. J Appl Psychol. 2005 Mar; 90(2):295-305.

31 Van Kleef GA. How emotions regulate social life: the emotions as social information (EASI) model. Curr Dir Psychol Sci. 2009;18(3):1848.

32 Van Kleef GA, De Dreu CK, Manstead AS. An interpersonal approach to emotion in social decision making: The emotions as social information model. Adv Exp Soc Psychol. 2010; 42:45-96.

33 Barsade SG, Gibson DE. Why does affect matter in organizations? Acad Manage Perspect. 2007;21(1):36-59.

34 Kamstra MJ, Kramer LA, Levi MD. Winter blues: A SAD stock market cycle. Am Econ Rev. 2003;93(1):324-43.

35 Russel JA. A circumplex model of affect. J Pers Soc Psychol. 1980;39(6):1161-78.

36 Barrett LF. Discrete emotions or dimensions? The role of valence focus and arousal focus. Cogn Emotion. 1998;12(4):579-99. 
37 Ekman P. An argument for basic emotions. Cogn Emotion. 1992;6(3-4):169-200.

38 Izard CE. Basic emotions, relations among emotions, and emotion-cognition relations. Psychol Rev. 1992 Jul;99(3):561-5.

39 Larsen RJ, Diener E. Affect intensity as an individual difference characteristic: A review. J Res Pers. 1987;21(1):1-39.

40 Blanchette I, Richards A. The influence of affect on higher level cognition: A review of research on interpretation, judgement, decision making and reasoning. Cogn Emotion. 2010; 24(4):561-95.

41 Williams LA, DeSteno D. Pride and perseverance: the motivational role of pride. J Pers Soc Psychol. 2008 Jun;94(6):1007-17.

42 Loewenstein G, Lerner JS. The role of affect in decision making. In: Davidson RJ, Scherer KR, Goldsmith HH, editors. Handbook of affective sciences. Oxford: Oxford University Press; 2003. p. 619-42.

43 Gross JJ. Antecedent- and response-focused emotion regulation: divergent consequences for experience, expression, and physiology. J Pers Soc Psychol. 1998 Jan;74(1):224-37.

44 Putora PM, Oldenburg J. The Kafkaesque process of cancer diagnosis. J Clin Oncol. 2014 Apr;32(10):1087-8.
45 Gross JJ, Sheppes G, Urry HL. Cognition and emotion lecture at the 2010 SPSP Emotion Preconference: Emotion generation and emotion regulation: A distinction we should make (carefully). Cogn Emotion. 2011;25(5):76581.

46 Tamir M. What do people want to feel and why? Curr Dir Psychol Sci. 2009;18(2):101-5.

47 Mauss IB, Bunge SA, Gross JJ. Automatic emotion regulation. Soc Personal Psychol Compass. 2007;1(1):146-67.

48 Hochschild AR. The managed heart. Berkeley: University of California Press; 1983.

49 Morris JA, Feldman DC. The dimensions, antecedents, and consequences of emotional labor. Acad Manage Rev. 1996;21(4):986-1010.

50 Grandey AA. Emotion regulation in the workplace: a new way to conceptualize emotional labor. J Occup Health Psychol. 2000 Jan;5(1):95-110.

51 Hatfield E, Cacioppo JT, Rapson RL. Emotional contagion. Curr Dir Psychol Sci. 1993; 2(3):96-100.
52 Giordano FA, Welzel G, Siefert V, Jahnke L, Ganslandt T, Wenz F, et al. Digital follow-up and the perspective of patient-centered care in oncology: what's the PROblem? Oncology. 2018. DOI: $10.1159 / 000495294$.

53 Walsh S, de Jong EE, van Timmeren JE, Ibrahim A, Compter I, Peerlings J, et al. Decision Support Systems in Oncology. JCO Clin Cancer Inform. 2019 Feb;3(3):1-9.

54 Naqa IE, Kosorok MR, Jin J, Mierzwa M, Ten Haken RK. Prospects and challenges for clinical decision support in the era of big data. JCO Clin Cancer Inform. 2018;2(2):1-12.

55 Ahlbrandt J, Lablans M, Glocker K, StahlToyota S, Maier-Hein K, Maier-Hein L, et al. Modern information technology for cancer research: what's in IT for Me? An overview of technologies and approaches. Oncology. 2018. DOI: $10.1159 / 000493638$.

56 Bandaragoda T, Ranasinghe W, Adikari A, de Silva D, Lawrentschuk N, Alahakoon D, et al. The Patient-Reported Information Multidimensional Exploration (PRIME) Framework for Investigating Emotions and Other Factors of Prostate Cancer Patients with Low Intermediate Risk Based on Online Cancer Support Group Discussions. Ann Surg Oncol. 2018 Jun;25(6):1737-45. 